\title{
Analysis of the Element's Arrangement Structures in Discrete Numerical Sequences
}

\author{
Anton Epifanov \\ Institute of precision mechanics and control, Russian Academy of Sciences, Russia \\ Received December 15, 2019; Revised January 13, 2020; Accepted February 7, 2020
}

Copyright $\mathrm{C} 2020$ by authors, all rights reserved. Authors agree that this article remains permanently open access under the terms of the Creative Commons Attribution License 4.0 International License

\begin{abstract}
Paper contains the results of the analysis of the laws of functioning of discrete dynamical systems, as mathematical models of which, using the apparatus of geometric images of automatons, are used numerical sequences which interpreted as sequences of second coordinates of points of geometric images of automatons. The geometric images of the laws of the functioning of the automaton are reduced to numerical sequences and numerical graphs. The problem of constructing an estimate of the complexity of the structures of such sequences is considered. To analyze the structure of sequences, recurrence forms are used that give characteristics of the relative positions of elements in the sequence. The parameters of recurrent forms are considered, which characterize the lengths of the initial segments of sequences determined by recurrence forms of fixed orders, the number of changes of recurrent forms required to determine the entire sequence, the place of change of recurrence forms, etc. All these parameters are systematized into the special spectrum of dynamic parameters used for the recurrent determination of sequences, which is used as a means of constructing estimates of the complexity of sequences. In this paper, it also analyzes return sequences (for example, Fibonacci numbers), for the analysis of the properties of which characteristic sequences are used. The properties of sequences defining approximations of fundamental mathematical constants (number e, pi number, golden ratio, Euler constant, Catalan constant, values of Riemann zeta function, etc.) are studied. Complexity estimates are constructed for characteristic sequences that distinguish numbers with specific properties in a natural series, as well as for characteristic sequences that reflect combinations of the properties of numbers.
\end{abstract}

Keywords Discrete Dynamical System, Mathematical Model, Discrete Deterministic Automaton, Geometrical Image of Automata Mapping, Numerical Sequence, Complexity Estimation, Recurrent Form, Spectrum of Dynamical Parameters, Characteristic Sequence

\section{Introduction}

The study of the functioning of discrete systems is the subject of research by thousands of scientists around the world. The areas of application of the results are very extensive - managing the functioning of industrial production, transport management, genomic technologies, managing electric power networks, description of the dynamics of population development, and much more (see, for example, one of the most interesting monographs [8] written by James T. Sandefur, also see $[9,12,13,16])$.

The traditional used mathematical models of discrete systems are defined by symbolic structures: tables, graphs, matrices, logical equations, etc. These models are not suitable for use in the control and diagnostics of large-scale and complex discrete systems (see, for example $[9,10]$ ), due to the enormous dimension. In $[1,2]$, a new approach based on numerical structures by professor Tverdokhlebov V.A. was proposed and developed to set the laws of functioning of control objects and technical diagnostics, which are complex systems. This approach allows you to use powerful idealizations of classical continuous mathematics: infinitesimal, actual infinity, summation of infinite series, passage to the limit, etc.

The approach developed in $[1,2]$ consists in converting symbolic models of diagnostic objects (automatons tables, matrices, graphs, logical equations) into numerical structures - geometric images of the laws of the functioning of automata (graphs with numerical coordinates of points) and interpreting the diagnostic effect on the technical object diagnosis as an input signal of the automaton, and the observed reaction to the impact as the output signal of the automaton.

The proposed approach allows to set the laws of functioning by geometric figures, which in turn can be set analytically, combine diagnostic tools of various nature in 
a single form - an experiment with an automaton, use the classical methods of interpolation and extrapolation. The developed geometric image is a phase picture of the diagnostic object, in which sections show specific options for the functioning of the object - phase trajectories.

It was shown in [3] that diagnostic procedures of various kinds (testing, measuring physical parameters, visual inspection and observation of signals, etc.) can be combined into a single and general form of experimental procedures with automata. A significant drawback of the application of the theory of experiments with automata for complex systems diagnosing is the symbolic representation of the laws of functioning of the automaton (tables, graphs, matrices, logical equations), which is not applicable for the technical diagnosis of complex systems. Mathematical tools suitable for dealing with the dimension of the objects under consideration, namely actual infinity, continuity, infinitesimal values, passage to the limit, summation of infinite series, etc. in symbolic automaton models are absent. In order to include these tools in the models of complex objects of control and technical diagnostics, the definition of the laws of functioning of discrete deterministic dynamic systems by numerical mathematical structures - geometric images of the laws of functioning (see [1-2]) is developed. The geometric images of the laws of the functioning of the automaton are reduced to numerical sequences and numerical graphs. Based on this, in the technical diagnosis of complex objects, formal methods for recognizing geometric curves that are represented in a geometric way by the behavior of objects of diagnosis and recognition of numerical sequences that uniquely determine the geometric image can be used.

In the geometric approach, the following scheme is used to convert an automaton mapping into a numerical sequence developed in $[1,2]$. The automaton mapping $\rho_{s_{0}}=\bigcup_{p \in X^{*}}\left\{\left(p, \lambda\left(s_{0}, p\right)\right)\right\}$ for the initial automaton $A_{s_{0}}=(\mathrm{S}$, $\mathrm{X}, \mathrm{Y}, \delta, \lambda, \mathrm{s}_{0}$ ) is linearly ordered based on the introduction of the linear order $\omega_{1}$ on the set $X^{*}$. In accordance with the linear orders $\omega_{1}$ on the set $\mathrm{X}^{*}$ and $\omega_{2}$ on $\mathrm{Y}$ and consideration of the function $\lambda^{\prime}: S \times X^{*} \rightarrow Y$, where $\lambda^{\prime}\left(s_{0}, p\right)$ is the last letter of the output word $\lambda\left(s_{0}, p\right)$, pairs of the form $\left(p, \lambda^{\prime}\left(s_{0}, p\right)\right) \in \rho_{s_{0}}$ are replaced by pairs $\left(r_{l}(p)\right.$, $\left.r_{2}\left(\lambda^{\prime}\left(s_{0}, p\right)\right)\right)$, where $r_{l}(p)$ - is the number of the input word $p$ in the order of $\omega_{1}$, and $r_{2}\left(\lambda^{\prime}\left(s_{0}, p\right)\right)$ - is the number of the last letter of the output word $\lambda\left(s_{0}, p\right)$ in order of $\omega_{2}$. The resulting numerical form of a linearly ordered automaton mapping $\left(\rho_{s_{0}}, \omega\left(\omega_{1}, \omega_{2}\right)\right.$ for the initial automaton $A_{s_{0}}$ is a numerical graph, which, accordingly, will be denoted $\gamma_{s_{0}}$.

From the geometrical image $\gamma_{s_{0}}$ of the automaton $A_{s_{0}}$, a sequence of second coordinates of points of the geometrical image is distinguished, which one-to-one corresponds to the full geometrical image (for fixed order on the set $X^{*}$ and the quantity $m=|X|$ ). As a result, the laws of the functioning of the automaton and the specific processes of the functioning of the automaton (i.e. phase trajectories) turn out to be uniquely determined by the sequence of second coordinates of the points of the geometric image. This allows to consider an arbitrary sequence of elements from a finite set as a sequence of second coordinates of points of a geometric image and, therefore, as a specification of the laws of functioning of the automaton. This allows to reduce the analysis of the laws of functioning of automaton models of systems to the analysis of the properties of numerical sequences. This approach is somewhat related to the approach described in [11] (monograph written by W.M. Wonham).

\section{Methods: Recurrent Definition Dynamic Parameters as a Means of Sequence Structure Complexity Estimation}

In $[2,4]$, the concept of a spectrum was introduced and developed, which characterizes the structure of a sequence, which can be considered as a way of definition of a geometric image of the laws of functioning.

Let be $U=\left\{u_{1}, u_{2}, \ldots, u_{k}\right\}$ a finite set and $\xi$ a sequence of elements from the set $U: \xi=\langle u(1), u(2), \ldots, u(t), \ldots\rangle$. The sets of all finite sequences, all finite sequences of length $\mathrm{v}$ and infinite sequences of elements from the set $\mathrm{U}$ will be denoted by $U^{*}, U^{v}, U^{\infty}$, respectively. The spectrum $\Omega(\xi)$ of dynamic characteristics of the sequence $\xi \in U^{*} \cup U^{\infty}$ has a hierarchical structure consisting of the levels $\Omega(\xi)=$ $\left(\Omega_{0}(\xi), \Omega_{1}(\xi), \Omega_{2}(\xi), \Omega_{3}(\xi), \Omega_{4}(\xi)\right)$. Each specific implementation option (represented by parameter values) of any level $\Omega_{i}(\xi)$ defines a partition of each of the sets $U^{*}$, $U^{v}, U^{\infty}$ into subsets according to the coincidence properties of the characteristics corresponding to the level. We will consider subsets of such a partition as equivalence classes of sequences.

Definition 1. For any sequence $\bar{\xi} \in U^{v}$, the least order of the recurrent form that defines the sequence $\bar{\xi}$ will be denoted $m_{0}(\bar{\xi})$.

Definition 2. For any sequence $\bar{\xi} \in U^{v}$ and $m \in N^{+}$, where $1 \leq m \leq m_{0}(\bar{\xi})$, the longest length of the initial segment of the sequence $\bar{\xi}$ determined by the recurrence form of order $m$ will be denoted $d^{m}(\bar{\xi})$.

Definition 3. For any sequence $\bar{\xi} \in U^{v}$ and $m \in N^{+}$, where, $1 \leq m \leq|\bar{\xi}|-1$, the number of shifts of recurrent forms of order $m$ required in determining the sequence $\bar{\xi}$ 
will be denoted $r^{m}(\bar{\xi})$.

Definition 4. For any sequence $\bar{\xi} \in U^{v}$ and $m \in N^{+}$, where $1 \leq m \leq m_{0}(\bar{\xi})$ and $j$, where $1 \leq j \leq r^{m}(\bar{\xi})$, the length of the $j$-th segment in the definition of a sequence $\bar{\xi}$, will be denoted $d_{j}^{m}(\bar{\xi})$.

Using the introduced notation, we define the spectrum of parameters characterizing the sequence as the following structure:

$$
\begin{gathered}
\Omega_{0}(\bar{\xi})=\left\langle m_{0}(\bar{\xi})\right\rangle ; \\
\Omega_{1}(\bar{\xi})=\left\langle d^{1}(\bar{\xi}), d^{2}(\bar{\xi}), \ldots, d^{\alpha}(\bar{\xi})\right\rangle ; \\
\Omega_{2}(\bar{\xi})=\left\langle r^{1}(\bar{\xi}), r^{2}(\bar{\xi}), \ldots, r^{\alpha}(\bar{\xi})\right\rangle ; \\
\Omega_{3}(\bar{\xi})=\left\langle\Omega_{3}^{1}(\bar{\xi}), \Omega_{3}^{2}(\bar{\xi}), \ldots, \Omega_{3}^{\alpha}(\bar{\xi})\right\rangle \quad \text { where } \alpha=m_{0}(\bar{\xi})
\end{gathered}
$$

and $\Omega_{3}^{j}(\bar{\xi})=\left\langle d_{1}^{j}(\bar{\xi}), d_{2}^{j}(\bar{\xi}), \ldots, d_{n_{j}}^{j}(\bar{\xi})\right\rangle\left(n_{j}-\right.$ the number of the last segment in the definition of a sequence $\bar{\xi}$ as a sequence of segments defined by individual recurrent forms of order $j$ );

$$
\Omega_{4}(\bar{\xi})=\Theta\left(\Omega_{3}(\bar{\xi})\right) \text {, where } \Theta \text { is the operator of }
$$

replacing in $\Omega_{3}(\bar{\xi})$ the lengths of the segments by the weights of the used recurrent forms for determining the segments.

The fourth level $\Omega_{4}(\bar{\xi})$ of the spectrum $\Omega(\bar{\xi})$ adds to the characteristics in the previous levels an assessment of the complexity of the rules and the options for using the rules.

The construction of the spectrum on the basis of the hierarchical structure was made with the aim of successively deepening and expanding the characteristics of the properties of the sequence when moving from the previous level to the next. Such structure allows one to reduce the set of parameters used, if the goal of analysis is achieved, and to be limited to the corresponding initial fragments of the spectrum.

Spectrum levels are hierarchically constructed by adding new parameters to the spectrum. In this regard, the equivalence of sequences is represented through $t$ equivalences determined by the level $\Omega_{\mathrm{t}}, 0 \leq t \leq 3$.

In this paper, we study the properties of fundamental mathematical quantities using a spectrum of dynamic parameters, determine the specificity of the structure of the arrangement of elements in each sequence. We consider the set of integer sequences presented in The Encyclopedia of Integer Sequences (founder N. J. A. Sloane) [5].

From this set a subset $\Xi$ is selected, consisting of 100 sequences of length 50 that determine the approximations of fundamental mathematical quantities $(\pi, \mathrm{e}, \gamma, \varphi$, etc.). For each $\xi \in \Xi$ was constructed 10 characteristic sequences $\kappa_{\xi}^{0}, \kappa_{\xi}^{1}, \kappa_{\xi}^{2}, \ldots, \kappa_{\xi}^{9}$, that determine the structure of the arrangement of decimal digits in the sequence. Are constructed a set of characteristic sequences $\mathrm{K}=\bigcup_{\xi \in \Xi}\left\{\kappa_{\xi}^{0}, \kappa_{\xi}^{1}, \kappa_{\xi}^{2}, \ldots, \kappa_{\xi}^{9}\right\}$, i.e. $|\mathrm{K}|=10 \cdot|\Xi|$. For each sequence from the set $\mathrm{K}=\bigcup_{\xi \in \Xi}\left\{\kappa_{\xi}^{0}, \kappa_{\xi}^{1}, \kappa_{\xi}^{2}, \ldots, \kappa_{\xi}^{9}\right\}$ are constructed the levels of the spectrum $\Omega_{0}-\Omega_{3}$ and the set is divided into subclasses of equivalent sequences by the coincidence of the numerical values of the dynamic parameters at the levels of the spectrum $\Omega_{0}-\Omega_{3}$.

The results of constructing partitions $P_{0}, P_{1}, P_{2}, P_{3}$ of the set $\mathrm{K}$ into subclasses of equivalent sequences are shown in table 1. For each of the constructed partitions, the table shows the values of the following quantities: the number of subclasses in the partition, the minimum power of the subclass, and the maximum power of the subclass.

Table 1. Information about partitions $P_{0}, P_{1}, P_{2}, P_{3}$ of the set $\mathrm{K}$ of characteristic sequences according to the parameters of the spectrum $\Omega$ at the levels $\Omega_{0}-\Omega_{3}$

\begin{tabular}{|c|c|c|c|c|}
\hline Characteristic & $\boldsymbol{P}_{\mathbf{0}}$ & $\boldsymbol{P}_{\mathbf{1}}$ & $\boldsymbol{P}_{\mathbf{2}}$ & $\boldsymbol{P}_{\mathbf{3}}$ \\
\hline $\begin{array}{c}\text { The number of subclasses in the } \\
\text { partition }\end{array}$ & 44 & 847 & 982 & 982 \\
\hline Maximum Power Subclass & 96 & 12 & 11 & 11 \\
\hline Minimum Power Subclass & 1 & 1 & 1 & 1 \\
\hline
\end{tabular}

\section{Results: Research of Characteristic Sequences That Determine the Structure of the Arrangement of Elements in Fundamental Mathematical Sequences}

As a result of constructing for each sequence from the set of numerical values K of the spectrum levels $\Omega_{0}-\Omega_{3}$, the specific structure of the arrangement of elements in each sequence of the set $\Xi$ is noted.

Table 2 shows the structure of the classes of equivalent in terms of the third level indicators $\Omega_{3}$ of spectrum $\Omega$, with a power of more than 1. Based on the obtained spectrum, the set of characteristic sequences is divided into equivalence classes according to the coincidence of the spectrums. For example, binary characteristic sequences reflecting locations in the number $\pi$ digits 3 and 9 are 0 equivalent, also equivalent in terms of the zero level of the spectrum are characteristic sequences that highlight the location of the number 5 in $\pi$ and the number 2 in $\pi / 2$. The equivalence noted of the characteristic sequences $\kappa_{\pi}^{8}$ and $\kappa_{\pi / 2}^{1}$ indicators of the zero level of the spectrum. In the partition $P_{3}$ of the set $\mathrm{K}$ of characteristic sequences, the presence of two-element classes is noted, whose members reflect the location of identical elements in the sequences that determine the approximation of $\log (\sqrt{2 \pi})$ and $\zeta^{\prime}(0)$. Also in the partition $P_{3}$ there are two-element classes 
whose members reflect the arrangement of identical elements in the sequences that determine the approximation of the quantities $\sqrt{\pi}$ and $\Gamma(1 / 2)$, where $\Gamma(x)=\int_{0}^{\infty} t^{x-1} e^{-t} d t, x>0$. To explain this fact, it is possible to use the results of [7]. In [7] it was shown that the sequences that determine the approximation of the quantities $\sqrt{\pi}$ and $\Gamma(1 / 2)$ are $0,1,2$ and 3 - are equivalent, the same shows the equivalence in terms of the third level of the spectrum of sequences that specify the values of the quantities $\log (\sqrt{2 \pi})$ and $\zeta^{\prime}(0)$, where $\zeta(s)=\sum_{n=1}^{\infty} \frac{1}{n^{s}}, s>1$.

Table 2. Structure of classes in partition $P_{3}$ of the set $\mathrm{K}$ consisting of more than one element

\begin{tabular}{|c|c|}
\hline$\kappa_{\sqrt{e}}^{9}, \kappa_{\Gamma(1 / 4)}^{4}, \kappa_{\psi(2 / 3)}^{9}, \kappa_{z}^{2}, \kappa_{z}^{3}, \kappa_{z}^{4}$, & 11 \\
\hline$\kappa_{z}^{5}, \kappa_{z}^{6}, \kappa_{z}^{7}, \kappa_{z}^{8}, \kappa_{z}^{9}$ & 3 \\
\hline$\kappa_{\log (7)}^{6}, \kappa_{\log (\pi)}^{6}, \kappa_{\Gamma(1 / 3)}^{1}$ & 2 \\
\hline$\kappa_{\sqrt{\pi}}^{5}, \kappa_{\Gamma(1 / 2)}^{5}$ & 2 \\
\hline$\kappa_{\log (\sqrt{2 \pi})}^{9}, \kappa_{\zeta^{\prime}(0)}^{9}$ & 2 \\
\hline$\kappa_{\sqrt{\pi}}^{7}, \kappa_{\Gamma(1 / 2)}^{7}$ & 2 \\
\hline$\kappa_{z}^{0}, \kappa_{z}^{1}$ & 2 \\
\hline$\kappa_{\sqrt{\pi}}^{9}, \kappa_{\Gamma(1 / 2)}^{9}$ & 2 \\
\hline$\kappa_{\log (\sqrt{2 \pi})}^{6}, \kappa_{\zeta^{\prime}(0)}^{6}$ & 2 \\
\hline$\kappa_{\zeta(2)}^{3}, \kappa_{\zeta(5)}^{2}$ & 2 \\
\hline$\kappa_{\sqrt{\pi}}^{8}, \kappa_{\Gamma(1 / 2)}^{8}$ & 2 \\
\hline$\kappa_{\log (\sqrt{2 \pi})}^{7}, \kappa_{\zeta^{\prime}(0)}^{7}, \kappa_{2 C_{2}}^{4}$ & 2 \\
\hline$\Gamma(1 / 2)$ & 2 \\
\hline & 2 \\
\hline & 2 \\
\hline
\end{tabular}

Table 2 Continued

\begin{tabular}{|c|c|}
\hline$\kappa_{\log (\sqrt{2 \pi})}^{8}, \kappa_{\zeta^{\prime}(0)}^{8}$ & 2 \\
\hline$\kappa_{1 / \sqrt{\pi}}^{1}, \kappa_{1 / \zeta(2)}^{9}$ & 2 \\
\hline$\kappa_{\sqrt{\pi}}^{2}, \kappa_{\Gamma(1 / 2)}^{2}$ & 2 \\
\hline$\kappa_{\log (\sqrt{2 \pi})}^{3}, \kappa_{\zeta^{\prime}(0)}^{3}$ & 2 \\
\hline$\kappa_{e^{-\pi}}^{5}, \kappa_{\zeta(1 / 2)}^{7}$ & 2 \\
\hline$\kappa_{\sqrt{\pi}}^{4}, \kappa_{\Gamma(1 / 2)}^{4}$ & 2 \\
\hline$\kappa_{\log (\sqrt{2 \pi})}^{5}, \kappa_{\zeta^{\prime}(0)}^{5}$ & 2 \\
\hline$\kappa_{e^{-\pi / 2}}^{2}, \kappa_{G / \pi}^{2}$ & 2 \\
\hline$\kappa_{\log (\sqrt{2 \pi})}^{0}, \kappa_{\zeta^{\prime}(0)}^{0}$ & 2 \\
\hline$\kappa_{\sqrt{\pi}}^{0}, \kappa_{\Gamma(1 / 2)}^{0}$ & 2 \\
\hline$\kappa_{\log (\sqrt{2 \pi})}^{1}, \kappa_{\zeta^{\prime}(0)}^{1}$ & 2 \\
\hline$\kappa_{\sqrt{\pi}}^{1}, \kappa_{\Gamma(1 / 2)}^{1}$ & 2 \\
\hline$\kappa_{\log (\sqrt{2 \pi})}^{2}, \kappa_{\zeta^{\prime}(0)}^{2}$ & 2 \\
\hline$\kappa_{\sqrt{\pi}}^{3}, \kappa_{\Gamma(1 / 2)}^{3}$ & 2 \\
\hline$\kappa_{\log (\sqrt{2 \pi})}^{4}, \kappa_{\zeta^{\prime}(0)}^{4}$ & 2 \\
\hline
\end{tabular}

For the problems of recognizing an automaton in a given family of automata, the condition for the existence of a solution to the problem is the absence of equivalent states for different automatons. This criterion is practically ineffective for large discrete systems. The developed by professor Tverdokhlebov V.A. geometric approach to defining the laws of functioning of discrete systems [1-4] allows to determine automatons even with a countable infinite number of states. The above criterion for the existence of an automaton recognition solution in this case loses its meaning. To replace it, it is proposed to use the considered spectrum. In this spectrum, all phase trajectories are systematically represented. Therefore, the properties of the trajectories are manifested in the properties of the spectrum. The described computational experiment shows 
on the example of analysis of fundamental mathematical sequences the possibility of classifying the laws of functioning according to the properties of the spectrum, the novelty of the approach in the classification, and the practical feasibility of the process of calculating the spectrums. Partially similar approaches to the analysis of discrete structures can be found, for example, in $[14,17]$.

\section{Research of Characteristic Sequences Reflecting the Properties of Natural Numbers}

The analysis of the OEIS sequence bank presented in [5] showed that in the bank a significant number of return sequences are present, i.e. sequences defined by the recurrence relation. In such sequences, each $m+1$ element is determined depending on $\mathrm{m}$ previous elements. Classical examples of such sequences are, for example, a series of Fibonacci numbers, Lukas numbers, Mersenne numbers, tribonacci numbers. Eduard Lukas introduced the concept of "generalized Fibonacci sequences", a special case of which are Fibonacci numbers and Luc numbers. Also in the OEIS sequence bank there is a significant number of sequences whose elements are positive numbers, interpreted, for example, as the number of steps in a certain algorithm, as a sequence of states, etc.

These sequences, as well as the main part of the return sequences extracted from OEIS, consist, as a rule, only from various elements. Obviously, if the sequence of numbers is explicitly analyzed using the spectrum of dynamic parameters, then the characteristics of the sequences at the levels $\Omega_{0}-\Omega_{3}$ of the spectrum $\Omega$ will turn out to be the same (except for a small number of sequences). The coincidence of characteristics is explained by the fact that in each of the considered sequences all the elements are different, i.e. to determine the entire sequence, the order of the recurrent form $m_{0}=1$ is sufficient. (A sequence of Fibonacci numbers does not have this property, since the first two elements are equal to 1 . To determine the Fibonacci sequence, $m_{0}=2$ is sufficient). In view of the described property of the return sequences, it is proposed to map a binary characteristic sequence to each sequence and to analyze many characteristic sequences using the spectrum $\Omega$.

A characteristic sequence $\chi(\xi)=\chi_{0}, \chi_{1}, \ldots, \chi_{t}$ selects numbers in the initial segment of a series of integers that belong to the return sequence $\xi$ to which it is associated,

i.e. $\chi_{i}=\left\{\begin{array}{l}0, \text { if } i \notin \xi \\ 1, \text { if } i \in \xi\end{array}\right.$ where $0 \leq i \leq t$.

where $\quad \Gamma(x)=\int_{0}^{\infty} t^{x-1} e^{-t} d t, x>0 \quad, \quad \psi(x)=\frac{\Gamma^{\prime}(x)}{\Gamma(x)}$, $\zeta(s)=\sum_{n=1}^{\infty} \frac{1}{n^{s}}, s>1, \quad \mathrm{C}_{2}=\prod_{p \geq 3}\left(1-\frac{1}{(p-1)^{2}}\right), \quad \mathrm{z}=<$ 1011011101111011111011111101111111011111111011 $1111>$.

Research of the set of characteristic sequences, including 248 elements, was carried out under the assumption that the specific properties of the return sequences extracted from OEIS (see [5]) are presented in the initial segments of characteristic sequences of length 100, i.e. $t=99$. For each of the 248 characteristic sequences, the levels $\Omega_{0}-\Omega_{3}$ of the spectrum $\Omega$ are constructed. By coincidence of the parameters values at the given levels, are constructed partitions $P_{0}, P_{1}, P_{2}, P_{3}$ of the set of characteristic sequences into classes of equivalent sequences. Accordingly, equivalent return sequences were determined. The specifics of partitions $P_{0}, P_{1}, P_{2}, P_{3}$ of the set of characteristic sequences are presented in table 3 .

Table 3. Information about partitions $P_{0}, P_{1}, P_{2}, P_{3}$ of the of characteristic sequences of return sequences

\begin{tabular}{|c|c|c|c|c|}
\hline Characteristic & $\boldsymbol{P}_{\mathbf{0}}$ & $\boldsymbol{P}_{\mathbf{1}}$ & $\boldsymbol{P}_{\mathbf{2}}$ & $\boldsymbol{P}_{\mathbf{3}}$ \\
\hline $\begin{array}{c}\text { The number of subclasses in the } \\
\text { partition }\end{array}$ & 44 & 847 & 982 & 982 \\
\hline $\begin{array}{c}\text { Maximum Power Subclass } \\
\text { Minimum Power Subclass }\end{array}$ & 96 & 12 & 11 & 11 \\
\hline
\end{tabular}

An analysis of the results shows that the partitions $P_{2}$ and $P_{3}$, constructed according to the indicators of the second and third levels of the spectrum, respectively, do not introduce changes in the structure of classes in the partition $P_{1}$. In the partitions $P_{1}, P_{2}$ and $P_{3}$ all classes are singleton.

Thus, the deeper properties of the return sequences, which are equivalent in terms of the levels $\Omega_{0}-\Omega_{3}$ of the spectrum $\Omega$, can be obtained by analyzing the corresponding characteristic sequence. An analysis of the results of the experiment showed that in the considered set of sequences there are 16 classes of sequences equivalent by zero level of the spectrum. 
Table 4. Class of equivalent sequences in values of parameters of level $\Omega_{0}$ of the spectrum $\Omega$, containing the characteristic sequence of the Fibonacci series

\begin{tabular}{|c|c|}
\hline $\begin{array}{l}\text { Number in } \\
\text { OEIS }\end{array}$ & $\begin{array}{l}\text { Description of the sequence, explicit form of the sequence, type of characteristic sequence } \\
\text { (starting length 100) }\end{array}$ \\
\hline A066819 & $\begin{array}{l}\text { Sum of the first n Sophie Germain primes. } \\
\xi=2,5,10,21,44,73,114,167,250,339,452,583,756,935,1126,1359,1598,1849,2130,2423,2782,3201,3682,4075 \\
\chi(\xi)=001001000010000000000100000000000000000000001000000000000000000000000000010000000000000000000 \\
0000000\end{array}$ \\
\hline A002512 & $\begin{array}{l}\text { Expansion of a modular function. } \\
\xi=1,2,5,10,22,40,75,130,230,382,636,1022,1645,2570,4002 \\
\chi(\xi)=011001000010000000000010000000000000000010000000000000000000000000000000000100000000000000000 \\
0000000\end{array}$ \\
\hline A110744 & $\begin{array}{l}\text { n divides } \mathrm{n} \text { described or is divisible by it. } \mathrm{n} \text { divides A045918(n) or A045918(n) divides } \mathrm{n} \\
\xi=1,2,5,10,22,44 \\
\chi(\xi)=011001000010000000000010000000000000000000001000000000000000000000000000000000000000000000000 \\
0000000\end{array}$ \\
\hline A075885 & $\begin{array}{l}\mathrm{a}(\mathrm{n})=1+\mathrm{n}+\mathrm{n}[\mathrm{n} / 2]+\mathrm{n}[\mathrm{n} / 2][\mathrm{n} / 3]+\mathrm{n}[\mathrm{n} / 2][\mathrm{n} / 3][\mathrm{n} / 4]+\ldots \text { where }[\mathrm{x}]=\text { floor }(\mathrm{x}) . \\
\xi=1,2,5,10,29,46,169,239,745,1450,4111,5182,27157 \\
\chi(\xi)=011001000010000000000000000001000000000000000010000000000000000000000000000000000000000000000 \\
0000000\end{array}$ \\
\hline A126204 & $\begin{array}{l}\text { Least prime representable as the arithmetic mean of two other primes in n different ways. } \\
\xi=2,5,11,17,37,53,89,71,101,179,137,193,233,257 \\
\chi(\xi)=001001000001000001000000000000000000010000000000000001000000000000000001000000000000000001000 \\
0000000\end{array}$ \\
\hline A000045 & $\begin{array}{l}\text { Fibonacci numbers: } \mathrm{F}(\mathrm{n})=\mathrm{F}(\mathrm{n}-1)+\mathrm{F}(\mathrm{n}-2), \mathrm{F}(0)=0, \mathrm{~F}(1)=1, \mathrm{~F}(2)=1, \\
\xi=1,1,2,3,5,8,13,21,34,55,89,144,233,377,610 \\
\chi(\xi)=011101001000010000000100000000000010000000000000000000010000000000000000000000000000000001000 \\
0000000\end{array}$ \\
\hline
\end{tabular}

Table 4 shows one of 16 classes of characteristic sequences equivalent at the zero level of the spectrum and the corresponding return sequences. The research of the properties of return sequences based on the analysis of their characteristic sequences using the spectrum $\Omega$ made it possible to reveal deeper properties of the return sequences themselves.

The results of the computational experiment show that, according to the quantitative values of the properties presented at the first, second, and third levels of the spectrum $\Omega$, in the considered set of characteristic sequences there are no pairs of equivalent sequences. Classification and complexity estimation in this case can be carried out according to the level indicators $\Omega_{0}$ of the spectrum $\Omega$.

\section{Research of Characteristic Sequences, Reflecting the Properties Combination}

As a result of the research of return sequences, a number of specific properties that these sequences possess are noted. For a more detailed research of the properties of the return sequences, an additional study was carried out, which consisted in the analysis using the spectrum of the set of characteristic sequences reflecting combinations of properties. In the general case, such characteristic sequences can be constructed on the basis of $n$ characteristic sequences. Elements of a characteristic sequence $\sigma\left(\chi\left(\xi_{1}\right), \ldots, \chi\left(\xi_{n}\right)\right)=\sigma_{1}, \sigma_{2}, \ldots, \sigma_{t} \quad$ constructed on the basis of $n$ characteristic sequences $\chi\left(\xi_{1}\right), \ldots, \chi\left(\xi_{n}\right)$, where $\xi_{1}, \ldots, \xi_{n}$ - the initial numerical sequences, are defined as follows:

$$
\begin{aligned}
& 0 \text {, if } \chi\left(\xi_{1}\right)=0 \& \chi\left(\xi_{2}\right)=0 \& \ldots \& \chi\left(\xi_{n}\right)=0 \text {, } \\
& 1 \text {, if } \chi\left(\xi_{1}\right)=0 \& \chi\left(\xi_{2}\right)=0 \& \ldots \& \chi\left(\xi_{n}\right)=1 \text {, } \\
& \sigma_{i}=\left\{2 \text {, if } \chi\left(\xi_{1}\right)=0 \& \chi\left(\xi_{2}\right)=0 \& \ldots \& \chi\left(\xi_{n-1}\right)=1 \& \chi\left(\xi_{n}\right)=0\right. \text {, } \\
& 2^{n}-1, \text { if } \chi\left(\xi_{1}\right)=1 \& \chi\left(\xi_{2}\right)=1 \& \ldots \& \chi\left(\xi_{n}\right)=1,
\end{aligned}
$$

where $\quad \chi(\xi)=\chi_{0}, \chi_{1}, \ldots, \chi_{t} \quad, \quad \chi_{i}=\left\{\begin{array}{ll}0, \text { if } & i \notin \xi \\ 1, \text { if } & i \in \xi\end{array}\right.$, $0 \leq i \leq t$.

Thus, for an arbitrary number of sequences, one characteristic sequence $\sigma=\sigma_{1}, \sigma_{2}, \ldots, \sigma_{t}$ can be constructed that uniquely determines the structure of the initial segments of the original sequences $\xi_{1}, \ldots, \xi_{n}$. Such characteristic sequence $\sigma=\sigma_{1}, \sigma_{2}, \ldots, \sigma_{t}$ can be constructed both from the original sequences $\xi_{1}, \ldots, \xi_{n}$ and from an arbitrary number of characteristic sequences $\chi\left(\xi_{1}\right), \ldots, \chi\left(\xi_{n}\right)$. In the paper for constructing sequences $\sigma=\sigma_{1}, \sigma_{2}, \ldots, \sigma_{t}$ both described methods are used.

The research included analysis using a spectrum of dynamic parameters of two sets of characteristic sequences $\Sigma_{2}$ and $\Sigma_{3}$. A number of return sequences (and sequences consisting of only different elements) $\Xi=\left\{\xi_{1}, \ldots, \xi_{100}\right\}$ were extracted from the OEIS sequence bank. The set $\Sigma_{2}$ consists of 10000 characteristic sequences constructed on 
the basis of return sequences from the set $\Xi$.

Each sequence $\sigma \in \Sigma_{2}, \sigma=\sigma_{1}, \sigma_{2}, \ldots, \sigma_{t}$, is built on the basis of two sequences $\xi_{i_{1}}, \xi_{i_{2}} \in \Xi$, i.e. $\sigma_{i}=\left\{\begin{array}{l}0, \text { if } i \notin \xi_{i_{1}} \& i \notin \xi_{i_{2}}, \\ 1, \text { if } i \notin \xi_{i_{1}} \& i \in \xi_{i_{2}}, \\ 2, \text { if } i \in \xi_{i_{1}} \& i \notin \xi_{i_{2}}, \\ 3 \text {, if } i \in \xi_{i_{1}} \& i \in \xi_{i_{2}},\end{array}\right.$ where $1 \leq i \leq 100$.

For sequences of set $\Sigma_{2}$ are constructed spectrums. Then, by the coincidence of the quantitative values at the levels $\Omega_{0}-\Omega_{3}$ of the spectrum $\Omega$, partitions of the set $P_{0}, P_{1}$, $P_{2}, P_{3}$ of the set $\Sigma_{2}$ into classes of equivalent sequences are constructed. The properties of partitions $P_{0}, P_{1}, P_{2}, P_{3}$ of the set are presented in table 5. The analysis of the results showed that a significant increase (more than 50 times) in the number of classes of equivalent sequences is observed when constructing the partition $P_{1}$ on the basis of the partition $P_{0}$ (the number of classes increases from 72 to 4073), while the maximum class power decreases almost 20 times in a partition (from 568 elements in a class to 30 elements).

Table 5. Information about partitions $P_{0}, P_{1}, P_{2}, P_{3}$ of the set $\Sigma_{2}$ of characteristic sequences according to the parameters of the spectrum $\Omega$ at the levels $\Omega_{0}-\Omega_{3}$

\begin{tabular}{|c|c|c|c|c|}
\hline Characteristic & $\boldsymbol{P}_{\mathbf{0}}$ & $\boldsymbol{P}_{\mathbf{1}}$ & $\boldsymbol{P}_{\mathbf{2}}$ & $\boldsymbol{P}_{\mathbf{3}}$ \\
\hline $\begin{array}{c}\text { The number of subclasses in the } \\
\text { partition }\end{array}$ & 72 & 4073 & 4890 & 4929 \\
\hline $\begin{array}{c}\text { Maximum Power Subclass } \\
\text { Minimum Power Subclass }\end{array}$ & 568 & 30 & 6 & 6 \\
\hline
\end{tabular}

Partition $P_{2}$ increases the number of classes to 4890 , while a 5 -fold decrease in the value of the maximum class power in the partition is observed. Partitioning $P_{3}$ increases the number of classes to 4929 .

In addition, for each sequence $\sigma \in \Sigma_{2}$, the value of the characteristic $\theta=\frac{m_{0} \cdot k}{n^{m_{0}-1}}$ is calculated, where $k$ is the number of characters in the sequence generated by applying the recurrence form $F$. By coincidence of the value of the characteristic $\theta$, a partition of $P_{\theta}$ into classes of sequences of equivalent complexity is carried out. The partition $P_{\theta}$ coincides with the partition $P_{0}$. The coincidence of the partitions $P_{\theta}$ and $P_{0}$ is explained by the fact that only those sequences for which the value of the characteristic $\theta$ coincides in one class of equivalent sequences in the partition $P_{0}$. (This is explained by the fact that, inside an arbitrary class in partition $P_{0}$, the value of the minimum order quantity of the recurrent form necessary to determine the sequence is the same for all elements of the class, the values of $k$ and $n$ also have the same values for all elements of the class: $k=10, n=4)$. The maximum value of the characteristic is $\theta=0.0038147$, the minimum is $2.34911 \mathrm{e}-$ 50 .

The set $\Sigma_{3}$ consists of 8000 sequences of length 100 . Each element of the set $\Sigma_{3}$ is construct on the basis of three characteristic sequences $\kappa_{1}^{i_{1}}(\xi), \kappa_{2}^{i_{2}}(\xi), \kappa_{3}^{i_{3}}(\xi) \in \mathrm{K}$ where $i_{1}, i_{2}, i_{3} \in\{0,1,2, \ldots, 9\}, \xi \in\{\pi, e\}$. The set $\mathrm{K}$ consists of 20 characteristic sequences of length 100 , reflecting the location of decimal digits in the sequence structure, specifying approximations of the fundamental mathematical quantities $\pi$ and $e$, i.e. $\mathrm{K}=\left\{\kappa_{\pi}^{0}, \kappa_{\pi}^{1}, \kappa_{\pi}^{2}, \kappa_{\pi}^{3}, \kappa_{\pi}^{4}, \kappa_{\pi}^{5}, \kappa_{\pi}^{6}, \kappa_{\pi}^{7}, \kappa_{\pi}^{8}, \kappa_{\pi}^{9}, \kappa_{e}^{0}, \kappa_{e}^{1}, \kappa_{e}^{2}, \kappa_{e}^{3}\right.$, $\left.\kappa_{e}^{4}, \kappa_{e}^{5}, \kappa_{e}^{6}, \kappa_{e}^{7}, \kappa_{e}^{8}, \kappa_{e}^{9}\right\}$. Elements of a sequence $\sigma=\sigma_{1}, \sigma_{2}, \ldots, \sigma_{t}$ from a set are defined as follows:

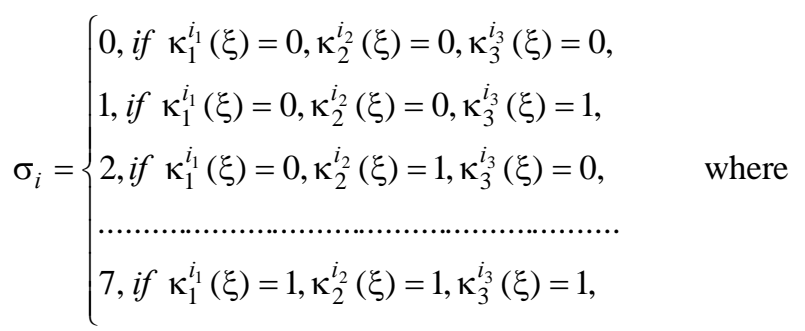

$i_{1}, i_{2}, i_{3} \in\{0,1,2, \ldots, 9\}, \xi \in\{\pi, e\}$. For sequences of set $\Sigma_{3}$ are constructed indicators of spectrum. Further, by the coincidence of the quantitative values at the levels $\Omega_{0}-\Omega_{3}$ of the spectrum $\Omega$, partitions of the set $P_{0}, P_{1}, P_{2}, P_{3}$ of the set are constructed into classes of sequences of equivalent structure elements. The properties of partitions $P_{0}, P_{1}, P_{2}$, $P_{3}$ of the set are presented in table 6 . An analysis of the results shows that a significant increase (more than 40 times) in the number of classes of equivalent sequences is observed when constructing the partition $P_{1}$ (the number of classes increases from 28 to 1220), while the value of the maximum class power in the partition decreases by more than 30 times (from 1248 elements in the class to 38 elements).

Table 6. Information about partitions $P_{0}, P_{1}, P_{2}, P_{3}$ of the set $\Sigma_{3}$

\begin{tabular}{|c|c|c|c|c|}
\hline Characteristic & $\boldsymbol{P}_{\mathbf{0}}$ & $\boldsymbol{P}_{\mathbf{1}}$ & $\boldsymbol{P}_{\mathbf{2}}$ & $\boldsymbol{P}_{\mathbf{3}}$ \\
\hline $\begin{array}{c}\text { The number of subclasses in the } \\
\text { partition }\end{array}$ & 28 & 1220 & 1350 & 1350 \\
\hline Maximum Power Subclass & 1248 & 38 & 6 & 6 \\
\hline Minimum Power Subclass & 1 & 1 & 1 & 1 \\
\hline
\end{tabular}

Partition $P_{2}$ increases the number of classes to 1350 , while a decrease in 6 times the value of the maximum class power in the partition is observed. The partition $P_{3}$ does not change the structure of the partition $P_{2}$. Also, for each sequence $\sigma \in \Sigma_{3}$, the value of the characteristic $\theta=\frac{m_{0} \cdot k}{n^{m_{0}-1}}$ is calculated, where $\mathrm{k}$ is the number of characters in the sequence generated by applying the recurrence form $F$. By coincidence of the value of the characteristic $\theta$, a partition of $P_{\theta}$ into classes of sequences of equivalent complexity is carried out. The partition $P_{\theta}$ coincides with the partition $P_{0}$. (An explanation of the coincidence of the partitions $P_{\theta}$ and $P_{0}$ is similar to that given above for the set $\Sigma_{2}$ ). The maximum value of the characteristic is $\theta=0.0488281$, the minimum is $1.16609 \mathrm{e}-$ 33. 


\section{Discussions and Possible Directions for Further Research}

The results obtained in the paper reflect the subtler properties of numbers of the natural series by considering combinations of the properties of numbers. One of the important and open questions in both number theory and cryptography is the question of the structure of the distribution of prime numbers in natural numbers. For finding prime numbers from more than 100,000,000 and 1,000,000,000 decimal digits EFF (Electronic Frontier Foundation - a nonprofit human rights organization founded in July 1990 in the United States to protect the rights embodied in the Constitution and the Declaration of Independence in connection with the advent of new communication technologies) awarded cash prizes of $\$ 150,000$ and $\$ 250,000$, respectively. Earlier, the EFF has already awarded prizes for finding prime numbers from $1,000,000$ and 10,000,000 decimal digits. In this regard, one of the possible directions for the further research can be the construction and analysis of spectrums for characteristic sequences of large length, which distinguish prime numbers in a natural number, as well as characteristic sequences reflecting combinations of the properties of prime numbers, Mersenne numbers, Lucas numbers, Woodall (or Riesel) numbers, numbers Cullen, Proth numbers, Mills numbers, etc. Perhaps, in this direction it will be possible to identify new properties of prime numbers that are interest.

\section{Conclusions}

Using the new geometric approach allows to: set the laws of functioning by numerical equations, supplement partially defined models of complex systems using interpolation and extrapolation methods, analyze the functioning of the system at any time intervals arbitrarily remote from the start of operation. Spectrums express the fundamental relationships between the laws of functioning and their decompositions into algorithmic fragments of laws. Such fragments are implemented in the selected basis of recurrent forms. The spectrum characterizes all variants of the bases of recurrent forms, which allows the use of spectrums as characteristics of laws. The results presented in the article show the possibility of the practical use of the spectrum of dynamic parameters to determine the properties of the laws of functioning of discrete deterministic dynamical systems based on the study of the properties of numerical sequences that one-to-one determine the laws of functioning.

\section{REFERENCES}

[1] V.A. Tverdokhlebov, The geometrical images of laws of functioning of finite state machines, Science book, Saratov, 2008.

[2] V.A. Tverdokhlebov, The geometrical images of the finite state machines, Proceedings of Saratov State University (a New series), Vol.5, Issue 1, 141-153, 2005.

[3] V.A. Tverdokhlebov, Geometrical approach to technical diagnosing of automatons, Proceeding of IEEE East-West Design \& Test Symposium (EWDTS'2011). Kharkov National University of Radioelectronics, 240-243, 2011.

[4] V.A. Tverdokhlebov, Complexity estimation of movement control by given route, Control science, No.5, 69-73, 2009.

[5] The On-Line Encyclopedia of Integer Sequences, Online available from: https://oeis.org/

[6] S.A. Abramov, Lectures on the complexity of algorithms, MCCNMO, Moscow, 2009.

[7] A.S. Epifanov, Models and methods of analysis and regularization methods of discrete dynamical systems functioning laws, Science book, Saratov, 2014.

[8] J.T. Sandefur, Discrete Dynamical Systems: Theory and Applications, Clarendon Press, Michigan, 1990.

[9] L.K. Carvalho, M.V. Moreira, J.C. Basilio, Diagnosability of intermittent sensor faults in discrete event systems, Automatica, 79, 315-325, 2017.

[10] O. Contant, S. Lafortune, D. Teneketzis, Diagnosis of intermittent faults, Discrete Event Dyn Syst, 14, 171-202, 2004.

[11] W.M. Wonham, Linear Multivariable Control: A Geometric Approach. Third ed. (revised), Springer-Verlag, New York, 1985.

[12] R.-Y. Zhang, K. Cai, Y. Gan, Z. Wang, W.M. Wonham. Supervision localization of timed discrete-event systems. Automatica, 49 (9), 2786-2794, 2013.

[13] D.Sears, K.Rudie, Computing observers from observation policies in discrete-event systems. Discrete Event Dyn. Syst. 28, No. 4, 509-537, 2018

[14] W.Wang, Online minimization of sensor activation for supervisory control, Automatica, 73, 8-14, 2016.

[15] M.Voráček, B.Melichar, Searching for regularities in generalized strings using finite automata, ICNAAM 2005. International conference on numerical analysis and applied mathematics 2005. Official conference of the European Society of Computational Methods in Sciences and Engineering (ESCMSE), Rhodes, Greek, September 16-20, 2005. 809-812, 2005.

[16] K. Mischaikow, M. Mrozek, Conley Index, Chapter 9 in Handbook of Dynamical Systems, vol. 2, 393-460. Elsevier, Amsterdam, 2002.

[17] F. Weilandt, The Conley index for discrete dynamical systems and the mapping torus, J. Appl. Comput. Topol. 3, No. 1-2, 119-138, 2019. 